\title{
Liver iron excess in patients with hepatocellular carcinoma developed on viral C cirrhosis
}

C Chapoutot, M Esslimani, Z Joomaye, J Ramos, P Perney, C Laurent, P Fabbro-Peray, D Larrey, J Domergue, F Blanc

\begin{abstract}
Background-Liver iron deposits are frequent in viral $C$ cirrhotic patients but their role is not well defined.

Aims-To investigate the effect of liver iron excess on the prevalence of hepatocellular carcinoma (HCC) in patients with viral $\mathrm{C}$ cirrhosis.

Methods-Hepatic iron was evaluated retrospectively using a semiquantitative method in liver biopsies of 104 viral $C$ cirrhotic patients, 48 with HCC and 56 controls (HCC free). Corrected total iron score $(0-60)$ was defined by the sum of three scores: hepatocytic iron score (036 ), sinusoidal iron score $(0-12)$, and portal iron score (0-12), multiplied by $3 / 3,2 / 3$, or $1 / 3$ according to the heterogeneous iron localisation in the nodules.

Results-After adjustment for known risk factors for HCC, regression analysis showed that iron deposits (corrected total iron score $>0$ ) were more frequent in HCC patients than in controls (odds ratio 4.94; 95\% confidence interval 1.59-15.32; $p=0.0056$ ). The median of corrected total iron score was significantly higher in HCC patients than in controls (odds ratio 1.092; 95\% confidence interval 1.01-1.13; $\mathrm{p}=0.021)$. This liver iron overload was sinusoidal (odds ratio $5.2 ; 95 \%$ confidence interval 1.82-15.11; $p=0.0022$ ).

Conclusions-Liver iron deposition was more frequent and more important in viral C cirrhotic patients with HCC than in HCC free controls. Liver iron overload seems to contribute to the development of HCC in patients with viral $\mathbf{C}$ cirrhosis. (Gut 2000;46:711-714)
\end{abstract}

Keywords: liver; iron; viral C cirrhosis; hepatocellular carcinoma

Hepatitis $\mathrm{C}$ virus (HCV) infection is a major aetiologic factor for hepatocellular carcinoma (HCC). ${ }^{1}$ But not all patients with $\mathrm{HCV}$ infection develop HCC and much effort has been directed towards determining high risk patients. In most cases cirrhosis or at least advanced hepatic fibrosis is found. ${ }^{2}$ Several additional risk factors for HCC have been identified in this population of viral $\mathrm{C}$ cirrhotic patients: male sex, increasing age, and chronic co-infection with hepatitis B virus. ${ }^{13-5}$ However, the mechanism by which HCV leads to $\mathrm{HCC}$ is not known. Moderate liver iron overload is frequent in $\mathrm{HCV}$ patients. ${ }^{6-9}$ Experimental studies support the hypothesis that iron may have a carcinogenic effect facilitating the development of HCC. $^{10}$ In one recent case control study, it was suggested that hepatic iron overload may contribute to the development of HCC in cirrhotic or noncirrhotic patients. ${ }^{11}$ These observations are not well documented in viral C cirrhotic patients.

In view of these considerations, we have examined the influence of liver iron overload on the prevalence of HCC in patients with viral C cirrhosis. Liver iron deposits were graded semiquantitatively in cirrhotic patients who had developed HCC and in controls without HCC.

\section{Materials and methods}

SUBJECTS

The study was performed in 104 patients with histologically proved cirrhosis who attended the Department of Internal Medicine, Surgery $\mathrm{C}$ and Department of HepatoGastroenterology, University Hospital of Montpellier, between January 1991 and April 1998. Histological diagnosis of cirrhosis was based on internationally accepted criteria. ${ }^{12}$ All patients had antibodies to $\mathrm{HCV}$, as determined using a second or third generation enzyme linked immunosorbent assay. We reviewed retrospectively histological sections of nontumoral liver samples performed in two groups of patients. Forty eight patients had HCC confirmed by histological examination. The control group included 56 patients without HCC identified by a normal liver ultrasound and serum $\alpha$ fetoprotein level $<20 \mathrm{ng} / \mathrm{ml}$.

Exclusion criteria in the two groups were: liver sample less than $0.4 \mathrm{~cm}$ in length or multifragmented biopsies without portal septa; family history of haemochromatosis or classic clinical expression of the disease (skin pigmentation, cardiac failure, diabetes, hypogonadism, arthritis); haemolytic disease; porphyria cutanea tarda; body mass index $>25$; iterative blood transfusions; serological markers for hepatitis B virus infection:hepatitis B surface antigen, hepatitis B core antibody; antinuclear antibody titre $>1: 40$; positive assays for antismooth muscle antibody; antimitochondrial antibody; abnormal ceruloplasmin; and $\alpha_{1}$ antitrypsin phenotype. In addition, no patient was infected with the human immunodeficiency virus.

The following data were collected: age; sex; duration of infection by $\mathrm{HCV}$ (when contami-

Abbreviations used in this paper: HCC hepatocellular carcinoma; HCV, hepatitis C virus; HIS, hepatocytic iron score; SIS, sinusoidal iron score; PIS, portal iron score; TIS, total iron score; cTIS, corrected total iron score. 
Table 1 Characteristics of patients with HCC and controls

\begin{tabular}{llllll}
\hline & $n$ & Patients with HCC & \multirow{2}{*}{ Controls } & $p$ Value \\
\hline Age (y) (mean (SD) [range]) & 48 & $66.1(8.5)[46-83]$ & 56 & $59.1(10.5)[32-80]$ & 0.0004 \\
Sex ratio (M/F) & 48 & $40 / 8$ & 56 & $35 / 21$ & 0.018 \\
Chronic alcoholism (No (\%)) & 48 & $11(22.9)$ & 56 & $24(42.8)$ & 0.03 \\
Duration of illness (y) (median (range)) & 20 & $14.5(5.5-37)$ & 29 & $16(10-35)$ & NS \\
Child-Pugh class (No (\%)) & 48 & A 26(54.2) & 56 & A 38 (67.9) & NS \\
& & B 11(22.9) & & B 12(21.4) & \\
HCV RNA+ (No (\%)) & 35 & C 11(22.9) & $54(10.7)$ & $52(96.3)$ & NS \\
Large oesophagal varices (No (\%)) & 37 & $21(56.7)$ & 50 & $24(48)$ & NS \\
Interferon treatment (No (\%)) & 48 & $8(16.7)$ & 56 & $16(28.6)$ & NS \\
\hline
\end{tabular}

nation was very probable and a precise date; transfusion or drug addiction in the past year); alcohol intake, defined as consumption of more than $40 \mathrm{~g}$ of alcohol over more than 10 years; interferon alpha treatment; serum HCV RNA detected by nested reverse transcription-PCR; and oesophageal varices (small, grade I; large, grades II-III). The degree of impairment of liver function was estimated using the ChildPugh score based on serum albumin, bilirubin, prothrombin time, presence of ascites, and encephalopathy.

\section{PATHOLOGICAL DATA}

Liver samples were obtained by resection in 11 cases during surgical treatment of HCC. These biopsies were at least $1 \mathrm{~cm}$ from the HCC. In 93 cases the biopsies were performed before the diagnosis of HCC (median five years (range 1-10)) using a percutaneous method. Specimens were fixed in alcoholic Bouin's liquid, embedded in paraffin, cut into $5 \mu \mathrm{m}$ thick slices, and stained with haematoxylin-eosin, Masson's trichrome, Sirius red, and Perls' staining. Each biopsy was examined by the same liver pathologist (ME) with no previous knowledge of the corresponding group. Histological iron content was graded semiquantitatively according to Deugnier and colleagues ${ }^{13}$ in the three areas of the Rappaport lobule. Iron deposits were assessed using three different scores: hepatocytic iron score (HIS), 0-36; sinusoidal iron score (SIS), 0-12; and portal iron score (PIS), 0-12. The total iron score (TIS) $0-60$, was defined as the sum of these three scores (TIS=HIS+SIS+PIS). To quantify heterogeneous iron distribution, a coefficient of heterogeneity was evaluated (screening at $\times 4$ magnification) according to Turlin and colleagues. ${ }^{14}$ This coefficient of heterogeneity was $1 / 3$ when iron distribution was very heterogeneous (iron deposits in 1/3 of the nodules), 2/3 when iron distribution was heterogenous (iron deposits in $1 / 3$ to $2 / 3$ of the nodules), and $3 / 3$ when liver siderosis was homogeneously distributed (iron deposits in more than $2 / 3$ of the nodules). Liver siderosis was finally quantified by the corrected total iron score: $c$ TIS $=$ TIS $\times$ coefficient of heterogeneity. Large liver cell dysplasia was screened for in all samples according to the morphological criteria proposed by Anthony and colleagues. ${ }^{15}$

STATISTICAL ANALYSIS

Results are expressed as mean (SD; range), median (range), or number (\%). Comparisons between groups were performed using the Student's $t$ test for quantitative variables with
Gaussian distribution and the Kruskal-Wallis test and Spearman's test for those with non-Gaussian distribution. The $\chi^{2}$ test or Fisher's exact test was used for comparison of qualitative variables. Iron scores and predictive factors of HCC were subsequently included in a stepwise forward logistic regression analysis to take into account confounding factors. A p value $<0.05$ was considered statistically significant.

\section{Results}

PATIENT CHARACTERISTICS

Patient data are shown in table 1. HCC patients were older than controls $(p=0.0004)$, were more often male $(\mathrm{p}=0.018)$, and had a history of chronic alcoholism more frequently $(p=0.03)$. Data collection was incomplete for three parameters (duration of illness, serum HCV RNA, and oesophageal varices).

HISTOLOGICAL ASSESSEMENT OF IRON

Liver samples were obtained by surgery in nine $(18.7 \%)$ HCC patients and in two $(3.6 \%)$ control patients $(\mathrm{p}=0.02)$; in the other cases they were obtained by percutaneous biopsy. Mean biopsy length was 11.8 (SD 7.1) $\mathrm{mm}$ in the HCC patients and 13.4 (SD 7.2) $\mathrm{mm}$ in controls $(p=0.3)$. Univariate analysis revealed that iron deposits (cTIS $>0$ ) were found histologically in $41(85.5 \%)$ HCC patients compared with $34(60.7 \%)$ controls $(p=0.005)$. cTIS, parenchymal (HIS), and mesenchymal (SIS, PIS) iron scores were higher in HCC patients than in controls (table 2). Localisation of sinusoidal iron deposits (SIS $>0$ ) was different between the two groups (table 3). Sinusoidal iron was exclusively at the periphery of nodules in $27.1 \%$ of HCC patients compared with $16 \%$ of controls, centronodular in $8.3 \%$ versus $21.5 \%$, and diffuse in $37.5 \%$ versus $12.5 \%(\mathrm{p}=0.002)$. In HCC patients, median cTIS values were comparable when the specimens were obtained by percutaneous biopsy or by surgery $(p=0.65)$. Similarly, the length of samples obtained by percutaneous biopsy had no influence on cTIS in either group

Table 2 Histological iron scores (median (range)) in HCC patients compared with controls

\begin{tabular}{llll}
\hline Score & $\begin{array}{l}\text { Patients with } \\
\text { HCC }(n=48)\end{array}$ & $\begin{array}{l}\text { Controls } \\
(n=56)\end{array}$ & $p$ Value \\
\hline cTIS & $7.6(0-31)$ & $2.3(0-23)$ & 0.0049 \\
HIS & $6(0-21)$ & $0(0-18)$ & 0.036 \\
SIS & $4(0-9)$ & $0.5(0-6)$ & 0.0002 \\
PIS & $1(0-5)$ & $0(0-6)$ & 0.046
\end{tabular}

cTIS, corrected total iron score; HIS, hepatocytic iron score; SIS, sinusoidal iron score; PIS, portal iron score. 
Table 3 Localisation of sinusoidal iron deposits

\begin{tabular}{llll}
\hline & $\begin{array}{l}\text { Patients with } \\
\text { HCC (\%) } \\
(n=48)\end{array}$ & $\begin{array}{l}\text { Controls (\%) } \\
(n=56)\end{array}$ & p Value \\
\hline No iron deposit & 27.1 & 50 & \\
Perinodular & 27.1 & 16 & 0.002 \\
Centronodular & 8.3 & 21.5 & \\
Diffuse & 37.5 & 12.5 & \\
\hline
\end{tabular}

Table 4 Significance of variables for prediction of hepatocellular carcinoma from multivariate analysis

\begin{tabular}{|c|c|c|c|}
\hline Variable & Risk ratio & $\begin{array}{l}95 \% \text { confidence } \\
\text { interval }\end{array}$ & $p$ Value \\
\hline Age $>62.5$ & 7.65 & $2.87-20.39$ & 0.0001 \\
\hline cTIS & 4.94 & $1.59-15.32$ & 0.0056 \\
\hline Sex (male) & 3.63 & $1.20-10.98$ & 0.022 \\
\hline
\end{tabular}

cTIS, corrected total iron score. In this model cTIS was entered by class $(0$ or $>0)$.

(rho $=0.27, \mathrm{p}=0.09$ in HCC patients and rho $=0.035, \mathrm{p}=0.79$ in controls). Alcohol consumption had no influence on cTIS $(\mathrm{p}=0.7)$, HIS $(p=0.9)$, PIS $(p=0.5)$, or SIS $(p=0.3)$. Duration of HCV infection, known in 49 patients, had no influence on cTIS $(\mathrm{p}=0.18)$.

Large liver cell dysplasia was observed in 18 $(37.5 \%)$ HCC patients and in $16(28.6 \%)$ controls $(\mathrm{p}=0.33)$.

Eight parameters were introduced into the logistic model according to a stepwise forward procedure: age, sex, treatment by interferon, consumption of alcohol, Child-Pugh class, oesophageal varices, large liver cell dysplasia, and cTIS. The analysis indicated that the prevalence of iron deposits (cTIS >0) in patients with HCC was significantly greater than that in controls (table 4). In a second model, median cTIS was introduced with the other variables. The cTIS was higher in HCC patients than in controls $(\mathrm{p}=0.021$; odds ratio (OR) $1.092 ; 95 \%$ confidence interval 1.011.13). When the three scores were introduced into the model, SIS was significantly higher in HCC patients than in controls $(\mathrm{p}=0.0022$; OR $5.2 ; 95 \%$ confidence interval 1.82-15.11), while the two groups did not differ in HIS or PIS. In the three models, male sex and age were the other significant predictive risk factors of HCC (table 4).

\section{Discussion}

Hepatitis $\mathrm{C}$ virus infection is recognised as a major risk factor for HCC but the precise mechanism of carcinogenesis is not known. ${ }^{1}$ RNA of HCV does not seem to be integrated into the host $\mathrm{DNA}^{16}$ contrary to hepatitis $\mathrm{B}$ virus DNA, ${ }^{17}$ which suggests an indirect carcinogenic influence. Liver iron could be a possible additional risk factor for HCC. ${ }^{10}$

Our study shows for the first time in a large group of patients with viral $\mathrm{C}$ cirrhosis that histological liver iron deposits were more frequent and more important in patients who had developed HCC compared with controls without HCC. Multivariate analysis showed that iron overload was correlated with HCC, independent of the existence of other known risk factors, and that iron excess was sinusoidal. The two other parameters with independent predictive value for HCC were age and male gender, as in previous studies. ${ }^{1-5}$ In a previous investigation, the hepatic iron index (liver iron concentration/age) in 20 patients with HCC developed on cirrhosis was significantly higher than in controls ( 24 cirrhotic patients without HCC), ${ }^{11}$ but the causes of cirrhosis were not well described, in particular exposure to hepatitis B or C viruses. In our series of patients, all HCC risk factors were analysed to determine precisely the role of iron. Coinfection with hepatitis $\mathrm{B}$ virus has been described as an additional aetiological factor for $\mathrm{HCC}^{3}$; hence these patients were excluded. The effect of HCV genotype $1 \mathrm{~b}$ has been scrutinised as another risk factor for HCC, ${ }^{18}{ }^{19}$ but three recent studies revealed no preferential role of individual $\mathrm{HCV}$ genotypes. ${ }^{20-22}$ In our series HCV genotyping was performed only in 32 patients and the influence of this variable was not analysed.

A diagnosis of haemochromatosis in our series cannot be strictly excluded because the C282Y mutation was not assessed. But no patient had a familial history of haemochromatosis, clinical signs of the disease, or had undergone venesection therapy. Moreover, contrary to the liver pathology seen in genetic haemochromatosis patients, iron excess was mild or moderate with a heterogeneous distribution, and was mainly located within mesenchymal cells.

Hepatic iron was estimated using a semiquantitative method rather than by direct measurement. We believe that the method used by Deugnier et al is the most reliable. There is a good correlation between results obtained by semiquantitative assessment and a quantitative method. ${ }^{13}$ In contrast, Ludwig and colleagues ${ }^{23}$ reported that in cases of mild hepatic iron abnormalities, iron quantitation may give false negative results, particularly in a liver with extensive fibrosis, but all patients in the present study had cirrhosis. The semiquantitative method also has the advantage that lobular and cellular distribution of iron can be assessed.

Our findings do not contradict previous studies showing that many patients with viral C cirrhosis have liver iron overload. Ludwig and colleagues $^{23}$ found positive iron stains in 18 of $43(41.9 \%)$ viral C cirrhotic patients using a different grading method. In our series, chronic alcohol use did not seem to have an effect on iron overload, in common with Piperno and colleagues' ${ }^{8}$ prospective study in $\mathrm{HCV}$ patients and in patients with haemochromatosis, ${ }^{13}$ but in contrast with another report. ${ }^{24}$ We believe that this difference may be explained by lack of an accurate method of identifying and quantifying precisely alcohol consumption.

The source of liver iron deposit is not clear. Heterozygosity for one of the two mutations of the HFE gene at position $282(\mathrm{C} 282 \mathrm{Y})$ or 63 (H63D) could play a role. ${ }^{95}$ But in other groups, heterozygosity for the HFE gene mutation had no influence on iron overload. ${ }^{26-28}$ A second hypothesis is that iron excess in the reticuloendothelial cells could come from damaged hepatocytes. Indeed, a significant relationship was found between the severity of histological activity and mesenchymal liver iron 
overload. ${ }^{26}{ }^{29}$ Finally, Deugnier et al have suggested that sinusoidal iron excess could reflect the duration of the disease in patients with haemochromatosis. ${ }^{13}$ In the present study, we found no significant correlation between iron deposits (cTIS) and duration of illness. But this information was obtained in only 49 $(47 \%)$ of 104 patients. Other studies are therefore necessary to clarify these hypotheses.

Whatever the source of sinusoidal iron (mutation of the $\mathrm{HFE}$ gene, reflection of disease duration, other causes), it seems to play a role in liver injuries. Experimental studies indicate that the cellular site of iron deposition in the liver is important for its carcinogenic potential. Dietary carbonyl iron used in an animal model to initiate the parenchymal iron deposition seen in early non-cirrhotic genetic haemochromatosis patients does not present any carcinogenic properties. ${ }^{30}$ Conversely, parenteral administration of iron dextran leads to reticuloendothelial cell iron deposition and has a direct role in hepatocarcinogenesis in the rat liver without cirrhosis. ${ }^{31}$ In our study, iron excess was mainly located within reticuloendothelial cells, and this is the main argument in favour of the role of iron deposits in hepatic carcinogenesis.

The mechanism by which increased liver iron may contribute directly to hepatocarcinogenesis still needs to be determined. One hypothesis is that iron could act by triggering the production of reactive oxygen species which may induce secondary lipid peroxidation, oxidative damage to proteins and nucleic acids, and finally initiate and promote carcinogenesis. ${ }^{1032}$

In the present study, we found an association between mildly increased hepatic iron stores and HCC in viral C cirrhotic patients, which could represent a cause-effect relationship. These results may have important implications if they are confirmed by other studies. Liver biopsy should not only be practised to confirm a diagnosis of cirrhosis but also to assess hepatic iron deposits. The second implication is that phlebotomy therapy could be proposed in these patients to reduce the risk of HCC.

1 Di Bisceglie AM. Hepatitis C and hepatocellular carcinoma. Hepatology 1997;3 (suppl 1):34-8.

$2 \mathrm{Di}$ Bisceglie AM, Simpson LH, Lotze MT, et al. Development of hepatocellular carcinoma among patients with chronic liver disease due to hepatitis $\mathrm{C}$ viral infection. f Clin Gastroenterol 1994;19:222-6.

3 Kew MC, Yu MC, Kedda M, et al. The relative roles of hepatitis $\mathrm{B}$ and $\mathrm{C}$ viruses in the etiology of hepatocellular carcinoma in southern African Blacks. Gastroenterology carcinoma in so

4 Ganne-Carrié N, Chastang C, Chapel F, et al. Predictive score for the development of hepatocellular carcinoma and additional value of liver large cell dysplasia in western patients with cirrhosis. Hepatology 1996;23:1112-18.

5 Brunetto MR, Oliveri F, Koehler M, et al. Effect of interferon- $\alpha$ on progression of cirrhosis to hepatocellular carcinoma: a retrospective cohort study. Lancet 1998;351: 1535-9.
6 Di Bisceglie AM, Axiotis CA, Hoofnagle JH, et al. Mesurement of iron status in patients with chronic hepatitis. Gasment of iron status in patients wit
troenterology 1992;102:2108-13.

7 Isomura T, Yano M, Hayashi H, et al. Excess iron in the liver of patients with chronic hepatitis C. F Clin Electron Microsc 1992;25:231-7

8 Piperno A, D'Alba R, Fargion S, et al. Liver iron concentration in viral hepatitis: a study of 98 patients. Eur $\mathcal{F}$ Gastroenterol Hepatol 1995;7:1203-8.

9 Smith BC, Grove J, Guzail MA, et al. Heterozygosity for hereditary hemochromatosis is associated with more fibrosis in chronic hepatitis C. Hepatology 1998;27:1695-9.

10 Deugnier Y, Turlin B, Loréal O. Iron and neoplasia. 7 Hepatol 1998;28:21-5.

11 Turlin B, Juguet F, Moirand R, et al. Increased liver iron stores in patients with hepatocellular carcinoma developed on a noncirrhotic liver. Hepatology 1995;22:446-50.

12 Anthony PP, Ishak K, Nayak NC, et al. The morphology of cirrhosis. F Clin Pathol 1978;3:395-414.

13 Deugnier Y, Loréal O, Turlin B, et al. Liver pathology in genetic hemochromatosis: a review of 135 homozygous cases and their bioclinical correlations. Gastroenterology 1992;102:2050-9.

14 Turlin B, Robert I, Maugendre S, et al. Quantification histologique des hépatosidéroses hémochromatosiques et
non hémochromatosiques. Ann Pathol 1997;5:329-32.

15 Anthony PP, Vogel CL, Barker LF. Liver cell dysplasia: a premalignant condition. $\mathcal{F}$ Clin Pathol 1973;26:217-23.

16 Fong T, Shindo M, Feinstone SM, et al. Detection of replicative intermediates of hepatitis C viral RNA in liver and serum of patients with chronic hepatitis C. F Clin Invest 1991;88:1058-60.

17 Shafritz DA, Shouval D, Sherman HI, et al. Integration of hepatitis B virus DNA into the genome of liver cells in chronic liver disease and hepatocellular carcinoma. $N$ Engl f Med 1981;305:1067-73.

18 Tanaka $\mathrm{K}$, Hirohata T, Ikematsu $\mathrm{H}$, et al. Hepatitis $\mathrm{C}$ virus infection and risk of hepatocellular carcinoma among Japanese: possible role of type $1 \mathrm{~b}$ (II) infection. $\mathrm{F}$ Natl Cancer Inst 1996;88:742-6.

19 Bruno S, Silini S, Crosignani A, et al. Hepatitis C virus genotypes and risk of hepatocellular carcinoma in cirrhosis a prospective study. Hepatology 1997;25:754-8.

20 Benvegnu L, Pontisso P, Cavalletto D, et al. Lack of correlation between hepatitis $C$ virus genotypes and clinical course of hepatitis C virus-related cirrhosis. Hepatology 1997;25:211-15.

21 Naoumov NV, Chokshi S, Metivier E, et al. Hepatitis C virus infection in the development of hepatocellular carcinoma in cirrhosis. F Hepatol 1997;27:331-6.

22 Kasahara A, Hayashi N, Mochizuki K, et al. Risk factors for hepatocellular carcinoma and its incidence after interferon treatment in patients with chronic hepatitis C. Hepatology treatment in patient

23 Ludwig J, Hashimoto E, Porayko MK, et al. Hemosiderosis in cirrhosis: a study of 447 native livers. Gastroenterology 1997;112:882-8.

24 Nishiguchi S, Kuroki T, Nakatani S, et al. Randomized trial of effects of interferon- $\alpha$ on incidence of hepatocellular carcinoma in chronic active hepatitis $\mathrm{C}$ with cirrhosis. Lancet 1995;346:1051-5.

25 Sampietro M, Boldorini R, Corbetta N, et al. Liver iron distribution, histological activity and mutations in the hemochromatosis gene (HFE) in chronic hepatitis C [abstract]. I Hepatol 1998;28 (suppl):98A.

26 Hézode C, Cazeneuve C, Coué O, et al. Liver iron overload in patients with chronic active hepatitis C. Prevalence, role of the hemochromatosis gene and relation with hepatic histological lesion [abstract]. F Hepatol 1998;28 (suppl):110A.

27 Curry GW, Thorburn D, Spooner RJ, et al. Heterozygosity for the C282Y mutation of the HFE gene is not associated with altered iron status or increased fibrosis in chronic hepatitis C (HCV) [abstract]. Hepatology 1998;28 (suppl): $451 \mathrm{~A}$.

28 Pirisi M, Toniutto P, Scott C, et al. HFE missense mutations, histological activity index and hemosiderosis in chronic hepatitis C [abstract]. Hepatology 1998;28 (suppl): 805A.

29 Kaji K, Nakanuma Y, Sasaki M, et al. Hemosiderin deposition in portal endothelial cells : a novel hepatic hemosiderosis frequent in chronic viral hepatitis B and C. Hum Pathol 1995;26:1080-5.

30 Stal P, Hultcrantz R, Moller L, et al. The effects of dietary iron on initiation and promotion in chemical hepatocariron on initiation and promotion in ch
cinogenesis. Hepatology 1995;21:221-8.

31 Carthew P, Nolan MB, Smith AG, et al. Iron promotes DEN initiated GST-P foci in rat liver. Carcinogenesis 1997;18: 599-603

32 Meneghini $\mathrm{H}$. Iron homeostasis, oxidative stress, and DNA damage. Free Radic Biol Med 1997;23:783-92. 